Original Research Article

\title{
Prescription pattern analysis of antibiotic use in a paediatric intensive care unit of a tertiary care teaching hospital in South India
}

\author{
Sindhu S.*, Syed Mohsin Ahmed
}

Department of Pharmacology, Mysore Medical College and Research Institute, Mysore, Karnataka, India

Received: 30 April 2018 Accepted: 04 May 2018

*Correspondence to:

Dr. Sindhu S.,

Email: dr.sindhusekar@ gmail.com

Copyright: (C) the author(s), publisher and licensee Medip Academy. This is an openaccess article distributed under the terms of the Creative Commons Attribution NonCommercial License, which permits unrestricted noncommercial use, distribution, and reproduction in any medium, provided the original work is properly cited.

\begin{abstract}
Background: Antibiotic resistance is rising to alarming levels that necessitates the evaluation of prescription patterns for the rational use of antibiotics. Hence this study was conducted to evaluate antibiotic use in a government run general hospital.

Methods: A prospective observational study was conducted to evaluate the rationality of antimicrobial prescription in a paediatric intensive care unit (PICU) of a government run tertiary care teaching hospital using the USAID indicators for rational use of antibiotics. Case records of 104 patients were documented and analysed.

Results: The most common antimicrobials prescribed were $3^{\text {rd }}$ generation Cephalosporins namely Ceftriaxone, followed by Aminoglycosides (Amikacin), Imipenams (Meropenam), Anti-Influenza Antiviral (Oseltamivir) and Oxazolidinones (Linezolid). A common trend of antibiotic overuse emerged due to paucity of resources to support decision making and choice of antibiotic. This led to patients being exposed to a high number of antibiotics with an associated increase in morbidity.

Conclusions: Antibiotic resistance would remain a challenge until systems for rapid, precise and low cost detection of the causative micro-organisms and antibiotic sensitivity are developed, surveillance systems are increased and antibiotic stewardship programs are enforced.
\end{abstract}

Keywords: Antibiotic resistance, Paediatric antibiotic overuse, Prescription pattern analysis, Rational antibiotic use

\section{INTRODUCTION}

Antibiotic resistance is dangerously rising to alarming levels in all parts of the world, putting the achievements of modern medicine at risk. The rapid spread and emergence of new resistance mechanisms threaten our ability to treat even the most common of infectious diseases such as pneumonia, tuberculosis, urinary tract and genital infections, fungal skin infections and sepsis. ${ }^{1}$

The emergence and spread of resistance is made worse by lack of or non-adherence to standard treatment guidelines and over-prescription of antibiotics by health workers; inappropriate self-medication of antibiotics without a prescription- often in inadequate dosage or for nonbacterial infections; and non-adherence to dosing regimens by the public; apart from non-human use of antimicrobials in veterinary care and agriculture. ${ }^{2}$

There has been a recent surge in efforts to rationalize the use of antimicrobials due to increasing antimicrobial resistance and a dwindling pipeline of new drugs under clinical development. ${ }^{3}$ These measures include increasing awareness, surveillance, research and investment in countering antimicrobial resistance. Among the key issues raised by the WHO is irrational prescription behaviour by health care workers. ${ }^{4}$ To address the problems of irrational 
use of drugs, the first step is to measure and document the drug use, identify problem areas, analyse it and understand the underlying causes. ${ }^{5}$

Hence a short term study of the prescription patterns was conducted in a Paediatric Intensive Care Unit (PICU) of a tertiary care, government run general hospital with the objectives of analysing the prescription pattern and determining the rationality of antimicrobial use.

\section{METHODS}

After obtaining Institutional Ethical Committee approval, a prospective observational study was conducted in the PICU of a tertiary care teaching hospital in South India for a period of 3 months. This was a typical government run general hospital in a limited resource setting where most antibiotics were available free of cost and the PICU had 8 beds.

All case records of patients who were admitted in the PICU for a minimum of 24 hours during a 3 month study period were considered for the study. Patients who were transferred to other departments and those who were discharged against medical advice were excluded from the study. The final analysis was done only for those patients who received at least one antimicrobial prescription. The definition of an antimicrobial was broad and included any antibacterial, anti-mycobacterial, antiviral, antifungal or any anti-parasitic according to the WHO ATC classification.

Demographic data such as subject's initials, age and gender were recorded. Subject's provisional diagnosis, relevant investigations, results of culture and antibiotic sensitivity, duration of stay, complete prescription for the entire duration of stay at the PICU and clinical outcomes were recorded in a pretested format. Care was taken to maintain confidentiality and anonymity of the subject's data.

The hospital formulary list relevant to the PICU and procurement cost of those drugs was obtained from the central drug store of the hospital. Average market price of the drug was used to calculate cost for such drugs that were not from the hospital formulary list.

Rationality of antimicrobial use was evaluated using USAID/WHO indicators for antimicrobial use in hospitals. ${ }^{6}$ Specific hospital indicators and indicators describing surgical prophylaxis were not included as surgical cases were not admitted to the PICU. The relevant indicators used were:

\section{Prescribing indicators}

- Percentage of hospitalizations with one or more antimicrobials prescribed.
- Average number of antimicrobials prescribed per hospitalization in which antimicrobials were prescribed.

- Percentage of antimicrobials prescribed consistent with the hospital formulary list.

- Average cost of antimicrobials prescribed per hospitalization in which antimicrobials were prescribed.

- Average duration of prescribed antimicrobial treatment.

- Percentage of patients with pneumonia who are prescribed antimicrobials in accordance with standard treatment guidelines.

- Percentage of antimicrobials prescribed by generic name.

\section{Patient care indicators}

- $\quad$ Percentage of doses of prescribed antimicrobials actually administered.

- Average duration of PICU stay of patients who receive antimicrobials.

\section{Supplemental indicator}

- Number of antimicrobial drug sensitivity tests reported per hospital admission with curative antimicrobials prescribed.

The data from this study was analysed with Descriptive statistics such as Mean, Standard deviation, Frequency, Percentage, and Proportions.

\section{RESULTS}

\section{Demographic background}

A total of 126 case records were analysed prospectively during the period of 3 months, from which the complete case details and prescriptions were recorded from 104 case records meeting the study criteria.

The age of the patients varied from 2 months to 16 years with an average age of 4.52 years. The maximum number of patients $(43.27 \%)$ were between 1 to 5 years old. $25 \%$ of the patients were below the age of 1 year. There was a total of 50 male and 54 female children. (Table 1).

Table 1: Demographic background.

\begin{tabular}{|lllll|}
\hline Age & Male & Female & Total & $\%$ \\
\hline Less than 1 & 16 & 10 & 26 & $25.00 \%$ \\
\hline 1 to 5 & 20 & 25 & 45 & $43.27 \%$ \\
\hline 6 to 10 & 6 & 9 & 15 & $14.42 \%$ \\
\hline $\begin{array}{l}\text { Greater than } \\
10\end{array}$ & 8 & 10 & 18 & $17.31 \%$ \\
\hline Total & 50 & 54 & 104 & $100.00 \%$ \\
\hline
\end{tabular}


The 5 most common causes for admission to the PICU were Pneumonia (39.18\%), Sepsis (11.34\%), CNS Infections (11.34\%), Seizure disorder (7.22\%) and Respiratory infections other than pneumonia (5.15\%).

\section{Prescription pattern}

At least one antimicrobial was prescribed to $93.27 \%$ $(\mathrm{N}=97)$ and these 97 cases were included in the final analysis.

A total of 25 different antimicrobials were used in the 97 patients. The most common antimicrobials used were Ceftriaxone $(24.51 \%$ of the total number of antimicrobials prescribed), Amikacin (17.83\%), Meropenam (8.91\%), Oseltamivir (7.52\%) and Linezolid (7.24\%). A similar pattern was seen in prescriptions of children less than 1 year.

Ceftriaxone was prescribed to $84.62 \%$ of the patients followed by Amikacin (61.5\%), Meropenam (30.8\%), Oseltamivir (26\%) and Linezolid (25\%). The most common antimicrobials prescribed with their ATC classification are described in Table 2.

Table 2: Antimicrobials prescribed with ATC classification.

\begin{tabular}{|llll|}
\hline Drugs & \% drugs & $\%$ patients & ATC \\
\hline Ceftriaxone & 24.51 & 84.62 & J01DD04 \\
\hline Amikacin & 17.83 & 61.54 & J01GB06 \\
\hline Meropenam & 8.91 & 30.77 & J01DH02 \\
\hline Linezolid & 7.24 & 25.00 & J01XX08 \\
\hline Oseltamivir & 7.52 & 25.96 & J05AH02 \\
\hline Vancomycin & 6.69 & 23.08 & J01XA01 \\
\hline Acyclovir & 5.01 & 17.31 & J05AB01 \\
\hline $\begin{array}{l}\text { Amoxicillin + } \\
\text { Clavulanic acid }\end{array}$ & 2.79 & 9.62 & J01CR02 \\
\hline Azithromycin & 2.23 & 7.69 & J01FA10 \\
\hline $\begin{array}{l}\text { Piperacillin }+ \\
\text { Tazobactam }\end{array}$ & 2.23 & 7.69 & J01CR05 \\
\hline
\end{tabular}

$\%$ Drugs - \% of total drugs prescribed

$\%$ Patients - \% of patients prescribed

ATC - Anatomical Therapeutic Chemical (ATC) Classification System

\section{USAID indicators}

\section{Prescribing indicators}

A total of 338 antimicrobials were prescribed to 97 patients and the average number of antimicrobials prescribed per patient was $3.48 \pm 1.95$ antimicrobials. Of the 97 patients, $58.65 \%$ of the patients were prescribed 3 or more antimicrobials and the maximum number antimicrobials prescribed per patient was 9 antimicrobials (Figure 1). $89 \%$ of the prescriptions for antimicrobials were consistent with the hospital formulary list. The generic name was used for $89.07 \%$ of the prescribed antimicrobials.
The average duration of prescribed antimicrobial treatment was $4.88 \pm 4.49$ days and ranged from 1 to 35 days. Linezolid was prescribed for 35 days in one of the case records initially via parenteral route and later via oral route.

\section{Table 3: USAID/WHO indicators for antimicrobial use in hospitals.}

\begin{tabular}{|c|c|c|}
\hline $\mathbf{A}$ & Prescribing indicators & Results \\
\hline 1 & $\begin{array}{l}\text { Percentage of hospitalizations with } \\
\text { one or more antimicrobials } \\
\text { prescribed }\end{array}$ & $93.27 \%$ \\
\hline 2 & $\begin{array}{l}\text { Average number of antimicrobials } \\
\text { prescribed per hospitalization in } \\
\text { which antimicrobials were } \\
\text { prescribed }\end{array}$ & $\begin{array}{l}3.48 \\
( \pm 1.95)\end{array}$ \\
\hline 3 & $\begin{array}{l}\text { Percentage of antimicrobials } \\
\text { prescribed consistent with the } \\
\text { hospital formulary list. }\end{array}$ & $89.07 \%$ \\
\hline 4 & $\begin{array}{l}\text { Average cost of antimicrobials } \\
\text { prescribed per hospitalization in } \\
\text { which antimicrobials were } \\
\text { prescribed. (INR) }\end{array}$ & $\begin{array}{l}414.78 \\
( \pm 502.61)\end{array}$ \\
\hline 5 & $\begin{array}{l}\text { Average duration of prescribed } \\
\text { antimicrobial treatment }\end{array}$ & $\begin{array}{l}4.88 \\
( \pm 4.49)\end{array}$ \\
\hline 6 & $\begin{array}{l}\text { Percentage of patients with } \\
\text { pneumonia who are prescribed } \\
\text { antimicrobials in accordance with } \\
\text { standard treatment guidelines }\end{array}$ & $34.21 \%$ \\
\hline 7 & $\begin{array}{l}\text { Percentage of antimicrobials } \\
\text { prescribed by generic name }\end{array}$ & $88.64 \%$ \\
\hline B & Patient care indicators & Results \\
\hline 8 & $\begin{array}{l}\text { Percentage of doses of prescribed } \\
\text { antimicrobials actually administered }\end{array}$ & $100 \%$ \\
\hline 9 & $\begin{array}{l}\text { Average duration of PICU stay of } \\
\text { patients who receive antimicrobials }\end{array}$ & $\begin{array}{l}6.91 \\
( \pm 6.4)\end{array}$ \\
\hline C & Supplemental indicator & Results \\
\hline 10 & $\begin{array}{l}\text { Number of antimicrobial drug } \\
\text { sensitivity tests reported per } \\
\text { hospital admission with curative } \\
\text { antimicrobials prescribed }\end{array}$ & 5.01 \\
\hline
\end{tabular}

The average cost (based on hospital procurement rates) of antimicrobial treatment per prescription was 414.78 \pm 502.61 INR and ranged from 15.85 INR to 2091.5 INR per prescription. The most expensive antibiotics were Meropenam, Vancomycin, Piperacillin-Tazobactum, Linezolid and Amoxi-clav in that order. Antibiotics contributed to $70 \%$ of the total cost of the medication expenses.

Pneumonia was the most common cause for admission to the PICU $(\mathrm{N}=35,39.18 \%)$. The Standard Treatment Guidelines (STG) for pneumonia was followed only in $34.19 \%$ of the cases. Most of the patients with pneumonia were treated empirically with Ceftriaxone $(92.11 \%)$ and Amikacin $(86.84 \%)$ as against the recommended Amoxi- 
Clav (18.42\%). The most common drugs prescribed were Ceftriaxone, Amikacin, Oseltamivir, Meropenam, and Linezolid (Figure 2).

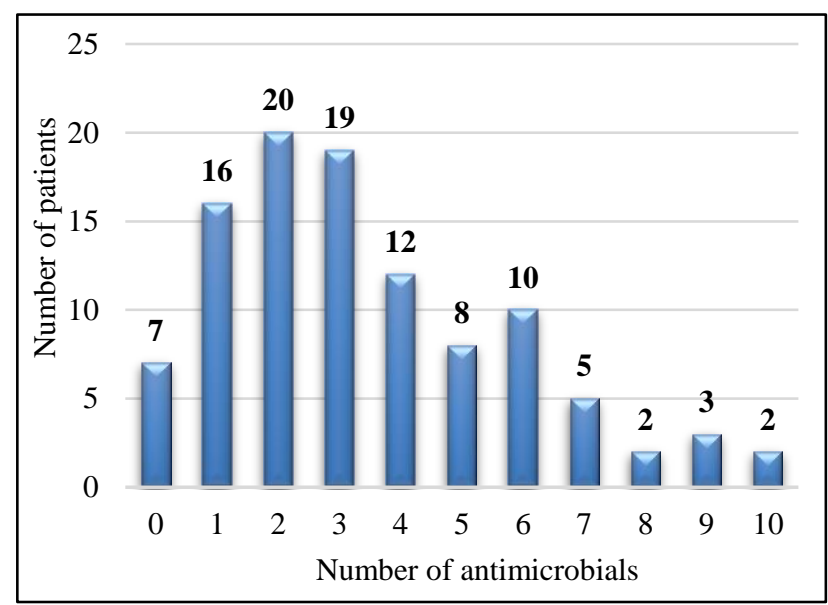

Figure 1: Number of antimicrobials prescribed.

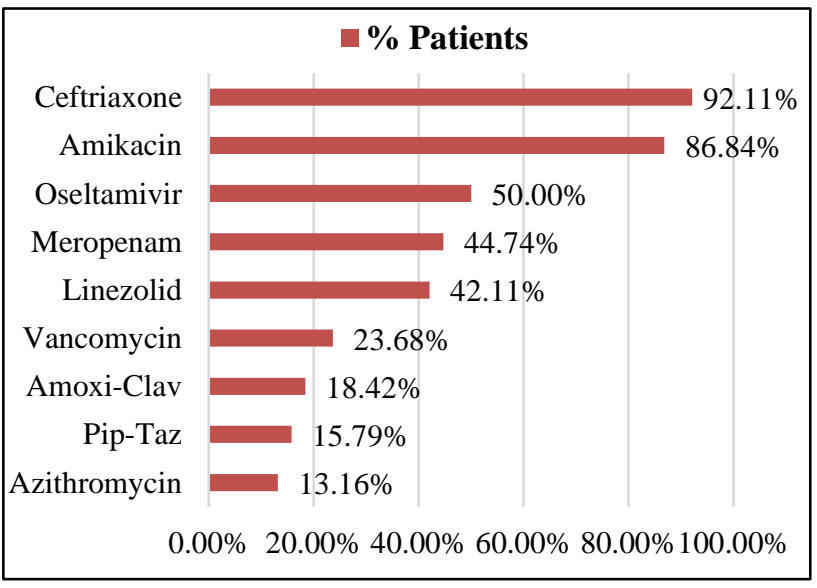

Figure 2: Antibiotic use in pneumonia.

\section{Patient care indicators}

$100 \%$ of the patients received the prescribed antimicrobials. This was based on treatment charts and nursing records. The average duration of stay of the patients in the PICU was $6.91 \pm 6.4$ days.

\section{Supplemental indicator}

Blood samples were sent for culture and sensitivity in almost all cases $(96 \%)$. Results of Culture and sensitivity was present in $22.68 \%$ of the case records but meaningful results that would help in the prescription of curative antimicrobials was obtained only in $5 \%$ of the cases.

\section{Prescription trends}

A common trend emerged where for most infections an empirical treatment of Ceftriaxone and Amikacin was initiated. If no response was seen by day 2 the antibiotics were changed to higher antibiotics such as Meropenam,
Linezolid, Vancomycin or Piperacillin- Tazobactam, on day 3. On further lack of response, the antibiotics were changed on day 5. Other antibiotics, Anti-Virals or AntiTB medications were prescribed on a patient to patient basis.

\section{Mortality}

There was no correlation between mortality to the duration of hospital stay. Mortality was highest in the initial 3-4 days of admission to PICU. But the \% mortality increased with number of drugs prescribed (Table 4).

Table 4: \% Mortality with number of antibiotics.

\begin{tabular}{|lllll|}
\hline $\begin{array}{l}\text { No. of } \\
\text { antibiotics }\end{array}$ & Death & Improved & Total & $\begin{array}{l}\% \\
\text { mortality }\end{array}$ \\
\hline 1 & 1 & 15 & 16 & $6.25 \%$ \\
\hline 2 & 3 & 17 & 20 & $15.00 \%$ \\
\hline 3 & 4 & 15 & 19 & $21.05 \%$ \\
\hline 4 & 5 & 10 & 15 & $33.33 \%$ \\
\hline 5 & 4 & 4 & 8 & $50.00 \%$ \\
\hline 6 & 6 & 6 & 12 & $50.00 \%$ \\
\hline 7 & 1 & 3 & 4 & $25.00 \%$ \\
\hline 8 & 0 & 2 & 2 & $0.00 \%$ \\
\hline 9 & 1 & 0 & 1 & $100.00 \%$ \\
\hline Total & 25 & 72 & 97 & $25.77 \%$ \\
\hline
\end{tabular}

\section{DISCUSSION}

In this study, $68 \%$ of the patients were below the age of 5 years, which is the most vulnerable age group in any population, which further underlines the importance of this study analysing the effects of drugs in such a vulnerable population.

The percentage of patients receiving at least one antimicrobial was high $(93.27 \%)$. But this is predictable considering firstly that they were acutely sick patients requiring intensive care, secondly the nature of illness comprising most commonly of pneumonia, CNS infections and sepsis and finally admission in the ICU may warrant prophylactic antibiotics in high risk patients. ${ }^{7}$

The most common antimicrobials prescribed were $3^{\text {rd }}$ generation Cephalosporins namely ceftriaxone, followed by Aminoglycosides (Amikacin), Imipenams (Meropenam), Anti-Influenza Antiviral (Oseltamivir) and Oxazolidinones (Linezolid). Similar trends were seen in other studies. ${ }^{8,9}$

The relatively high use of Anti-Influenza agents was because the period of the study coincided with an outbreak of H1N1 flu that occurred in the region between late 2016 and early 2017.

On an average, a patient was prescribed 3.5 drugs, but nearly $60 \%$ of the patients received 3 or more antimicrobials during their course of stay in the PICU. This 
could arbitrarily mean that most of the patients did not respond to the most common empirical combination of $3^{\text {rd }}$ generation Cephalosporin with an Aminoglycoside or Amoxi-clav. This seems to suggest resistance to these antibiotics, though definitive laboratory evidence was not available.

The duration of prescribed treatment averaged nearly 5 days. The recommended duration of treatment with linezolid is $10-28$ days. Longer duration of treatment is associated with haematological toxicity, optic neuropathy, peripheral neuropathy and lactic acidosis. ${ }^{10,11}$

As mortality was highest in the initial 3-4 days of admission, there was no correlation between mortality to the duration of hospital stay or number of antibiotics prescribed.

Community acquired pneumonia accounted for $15 \%$ of deaths in children globally in 2015 and is a common cause for childhood morbidity and mortality. ${ }^{9}$ Pneumonia was the most common cause for PICU admission in this study too and therefore warranted the need for further specific analysis. The National Treatment Guidelines for Antimicrobial Use in Infectious Diseases, 2016 - India recommend IV Amoxi-Clav or IV Ceftriaxone for pneumonia. ${ }^{12,13}$ STG was followed only in $34.19 \%$ of the cases. Most of the patients with pneumonia were treated empirically with a combination of Ceftriaxone $(92.11 \%)$ and Amikacin (86.84\%).

The usual trend was to prescribe antibiotics empirically usually a combination of Ceftriaxone and Amikacin to cover both gram positive and negative and sequentially switching to higher antibiotics if there was no response after 2 days of antibiotic administration. This was done until the results of culture and sensitivity were available.

But with meaningful culture and sensitivity results available in a mere $5 \%$ of the patients, physicians had little to base their treatment on and thus the patients continued to have their antibiotics escalated until a clinical response was seen. This led to patients receiving up to even 10 different antibiotics within a very short span of time. This overuse of antibiotics can have a serious implication in such a vulnerable population and can contribute to the development of resistance.

But in light of the critical nature of illness and paucity of resources, physicians are left with few alternatives. Thus, the need of the hour to combat resistance and improve patient safety is to develop sensitive and low cost solutions that could aid superior decision making and targeted therapy. ${ }^{14}$ This study also highlights the need to develop and implement strict antibiotic stewardship programmes in such government run general hospitals to check the unrestricted availability of higher antibiotics.

A recent WHO report confirms that the world is running out of antibiotics as there is a serious lack of new antibiotics under development. The report found very few potential treatment options for those antibiotic-resistant infections identified by WHO as posing the greatest threat to health, including drug-resistant tuberculosis. ${ }^{3}$

The overuse, underuse or misuse of antimicrobials results in wastage of scarce resources, widespread health hazards, unacceptable treatment outcomes and increased economic burden to patients and society in the form of costlier drugs, longer hospital stays and increased mortality. ${ }^{15}$

Hence there is an urgent need to change the way physicians prescribe and use antibiotics. Without behaviour change, we are headed for a post-antibiotic era, where once again minor infections and injuries can become fatal.

\section{ACKNOWLEDGEMENTS}

Authors would like to thank the physicians and nursing staff of the Department of Paediatrics for their guidance and support with data collection.

Funding: No funding sources

Conflict of interest: None declared

Ethical approval: The study was approved by the Institutional Ethics Committee

\section{REFERENCES}

1. World Health Organization. Antimicrobial resistance: global report on surveillance, 2014. Available at: http://apps.who.int/iris/handle/10665/112642.

Accessed 14 March 2018.

2. WHO. The pursuit of responsible use of medicines: sharing and learning from country experiences; 2012. WHO/EMP/MAR/2012.3

3. World Health Organization. Antibacterial agents in clinical development: an analysis of the antibacterial clinical development pipeline, including tuberculosis. 2017 Available at: http://www.who.int/medicines/news/2017/IAU_Anti bacterialAgentsClinicalDevelopment_webfinal_2017 _09_19.pdf. Accessed 27 April 2018.

4. World Health Organization (WHO). Antimicrobial resistance: global report on surveillance 2014. 2014; $1-256$ Available at: http://apps.who.int/iris/bitstream/10665/112642/1/97 89241564748_eng.pdf. Accessed 28 april 2018

5. Holloway K, Green T, World Health Organization. Drug and therapeutics committees: a practical guide. 2003. Available http://apps.who.int/medicinedocs/en/d/Js4882e/. Accessed 28 April 2018.

6. USAID. How to investigate antimicrobial use in health facilities: Selected indicators. February 2012. Available at: http://apps.who.int/medicinedocs/en/d/Js21031en/. Assessed 28 April 2018

7. Liberati A, D'Amico R, Pifferi S, Torri V, Brazzi L. Antibiotic prophylaxis to reduce respiratory tract 
infections and mortality in adults receiving intensive care. The Cochrane Library. 2004 Jan 1.

8. Laxminarayan R, Chaudhury RR. Antibiotic resistance in India: drivers and opportunities for action. PLoS Med. 2016;358:e1001974.

9. Baidya S, Hazra A, Datta S, Das AK. A study of antimicrobial use in children admitted to pediatric medicine ward of a tertiary care hospital. Indian $\mathbf{J}$ Pharmacol. 2017;49:10-5.

10. Garazzino S, Tovo PA. Clinical experience with linezolid in infants and children. J Antimicrob Chemother. 2011;66(4):23-41.

11. Vinh DC, Rubinstein E. Linezolid: a review of safety and tolerability. J Infect. 2009;59:59-74.

12. National Centre for Disease Control. National Treatment Guidelines for Antimicrobial Use in Infectious Diseases - India, 2016. Available at: http://pbhealth.gov.in/AMR_guideline7001495889.p df. Assessed on 21.8.2017
13. Haq IJ, Battersby AC, Eastham K, McKean M. Community acquired pneumonia in children. BMJ. 2017;356:686.

14. Das B, Chaudhuri S, Srivastava R, Nair GB, Ramamurthy T. Fostering research into antimicrobial resistance in India. BMJ. 2017;358:j3535.

15. Chandy SJ, Thomas K, Mathai E, Antonisamy B, Holloway KA, Stals by Lundborg C. Patterns of antibiotic use in the community and challenges of antibiotic surveillance in a lower-middle-income country setting: A repeated cross-sectional study in Vellore, South India. J Antimicrob Chemother. 2013;68:229-36.

Cite this article as: Sindhu S, Ahmed SM.

Prescription pattern analysis of antibiotic use in a paediatric intensive care unit of a tertiary care teaching hospital in South India. Int J Basic Clin Pharmacol 2018;7:1094-9. 